\section{(C) OPEN ACCESS}

\title{
Fixed-dose combination antihypertensives and risk of medication errors
}

\author{
Frank Moriarty, ${ }^{1}$ Kathleen Bennett, ${ }^{2}$ Tom Fahey ${ }^{1}$
}

\begin{abstract}
- Additional material is published online only. To view please visit the journal online (http://dx.doi.org/10.1136/ heartjnl-2018-313492).
\end{abstract}

${ }^{1}$ HRB Centre for Primary Care Research, Royal College of Surgeons in Ireland, Dublin, Ireland

${ }^{2}$ Division of Population Health Sciences, Royal College of Surgeons in Ireland, Dublin, Ireland

\section{Correspondence to} Dr Frank Moriarty, HRB Centre for Primary Care Research, Royal College of Surgeons in Ireland, Dublin 2, Ireland; frankmoriarty@rcsi.ie

Received 23 April 2018 Revised 10 July 2018 Accepted 10 July 2018 Published Online First 2 August 2018

\section{Linked}

- http://dx.doi.org/10.1136/ heartjnl-2018-313859

Check for updates

(C) Author(s) (or their employer(s)) 2019. Re-use permitted under CC BY-NC. No commercial re-use. See rights and permissions. Published by BMJ.

To cite: Moriarty $\mathrm{F}$ Bennett K, Fahey T. Heart 2019:105:204-209.

\begin{abstract}
Objective While fixed-dose combinations (FDC) can improve adherence, they may add complexity to the prescribing/dispensing process, potentially increasing risk of medication errors. This study aimed to determine if prescriptions for antihypertensive FDCs increase the risk of therapeutic duplication and drug-drug interactions (DDI).
\end{abstract}

Methods This retrospective observational study used administrative pharmacy claims data from the Irish Primary Care Reimbursement Service. Prescriptions dispensed to adults in 2015 were included if they contained an antihypertensive FDC, or the same drugs prescribed separately. The outcomes were therapeutic duplication and potentially serious DDI involving FDC drugs. Relative risk (RR) of these outcomes, adjusted for prescription and patient factors, was determined using generalised linear models with Poisson distributions and propensity score matching.

Results This study included 307833 FDC prescriptions (67.0\%) and 151632 separate component prescriptions. Half of patients prescribed FDCs were female with a mean age of 67.1 (SD 12.5) years and, compared with separate component prescriptions, FDCs were less often coprescribed with other cardiovascular medications. Therapeutic duplication occurred in $0.8 \%$ of prescriptions, most often involving calcium channel blockers, and $10.6 \%$ contained a DDI (most often amlodipine and simvastatin). The RR of therapeutic duplication on FDC prescriptions compared with separate component prescriptions was $1.46(95 \% \mathrm{Cl} 1.17$ to 1.83) and the adjusted RR was $2.06(95 \% \mathrm{Cl} 1.64$ to 2.60). For DDls, there was no significant difference between FDC and separate component prescriptions after confounder adjustment.

Conclusions This study found FDCs were associated with increased risk of duplication. When considering prescribing FDCs, this safety consideration should be weighed against potential benefits.

\section{INTRODUCTION}

Fixed-dose combinations (FDC), single medications or dosage forms that contain a combination of two or more active ingredients, are becoming increasingly common. ${ }^{12}$ They are most often used where multiple drugs may be required to treat/ control a condition, such as HIV/AIDS, diabetes, or hypertension, and polypills combining more diverse drug combinations (eg, for cardiovascular prevention) also exist. ${ }^{2}$ By replacing multiple treatments, they can reduce pill burden for patients and simplify medication regimens. For this reason,
FDCs can increase adherence compared with patients taking the equivalent drugs separately, ${ }^{2} 3$ and improve objective measures such as blood pressure. ${ }^{4}$ Combination therapy using multiple drugs acting on different therapeutic targets can produce synergistic effects and provide superior efficacy compared with monotherapy. Using multiple agents can also benefit tolerability where a combination allows for each drug to be used at lower doses than would be required if either drug was used alone, reducing the risk of adverse effects. ${ }^{5}$ Hypertension is one of the conditions where FDCs are used most commonly. ${ }^{35}$ Most patients require multiple antihypertensive drugs to meet blood pressure targets, and hence the greatest number of FDCs is in this therapeutic area. ${ }^{5}$ Hypertension is highly prevalent, particularly among older people, and is often comorbid with other conditions in this age group. ${ }^{6}$ Hence, FDCs may be particularly beneficial for such patients with high pill burdens for whom adherence to treatment may be challenging. ${ }^{7}$ Studies have demonstrated the advantages both in efficacy and safety of utilising multiple antihypertensives instead of a higher dose of a single agent, ${ }^{5}$ and there is evidence from a recent Cochrane review that initiating combination antihypertensive therapy rather than monotherapy may be advantageous. ${ }^{8}$

A disadvantage to FDCs is the 'fixed' nature of the dosing combinations may make dosage adjustment more difficult, leading to potential underdosing or overdosing. ${ }^{1}$ FDCs may reduce the ability to identify the cause of an adverse drug event relating to one of the drug ingredients. FDCs could also contribute to medication errors. They are frequently prescribed by brand name, ${ }^{9-11}$ and presence of multiple ingredients available in varying strengths within a single product adds complexity to the prescribing and dispensing process. $^{12}$ Medication errors cause a substantial burden of patient harm, morbidity and mortality ${ }^{13}$; however, the impact of FDCs on medication safety and prescribing quality has not been extensively evaluated to date. Therefore, the aim of this study is to determine if prescriptions for antihypertensive FDCs increase the risk of prescribing errors, namely therapeutic duplications and potentially serious drug-drug interactions (DDI), compared with the free combination (FC) ingredients prescribed separately.

\section{METHODS}

\section{Study design, setting and participants}

The Strengthening the Reporting of Observational Studies in Epidemiology statement has been used in the planning and reporting of this research. ${ }^{14}$ 
This is a retrospective cohort study using the Health Service Executive-Primary Care Reimbursement Service (HSE-PCRS) administrative pharmacy claims database. ${ }^{15}$ The analysis includes prescriptions dispensed to adults (aged 18 years and over) under the General Medical Service (GMS) scheme in Ireland in 2015. The GMS scheme is public health cover that provides free medical care and prescribed medications to about one-third of the general Irish population whose household income is below the eligibility threshold. It also covers the vast majority (approximately 95\%) of people aged 70 years and over, where a higher income threshold applies.

The unit of analysis was each prescription dispensing claim, and these were included if there was a dispensing for either of the following:

- An FDC of antihypertensive drugs where the components are also available to be prescribed separately.

- The same drugs as an FDC prescribed as separate components (or FC) in solid oral dosage form.

All such prescriptions dispensed to an adult on the GMS scheme during 2015 were included in the analysis. The relevant combinations available on the market, all of which were included in this study, are listed in online supplementary table S1. Permission was obtained from the HSE-PCRS to conduct this analysis. As the data were anonymised and results presented at group level, ethical approval was not required.

\section{Outcomes}

The primary outcome was therapeutic duplication involving a component of an included antihypertensive FDC, defined as two drugs from a therapeutic class (ACE inhibitors, angiotensin II receptor blockers (ARB), calcium channel blockers or beta blockers) on the same pharmacy claim (ie, dispensed from the one prescription). Products containing the same drug were not considered therapeutic duplication. The secondary outcome was the presence of a potentially serious DDI involving a component of an included antihypertensive FDC and another coprescribed medication. Interactions where the sole adverse effect relates to hypotension were excluded, as prescribers may have accounted for this interaction in the dose they prescribe and thus these are less likely to constitute medication errors. Interactions were considered potentially serious if they carry a 'black dot' in the British National Formulary (online supplementary table S2).

\section{Exposure and potential confounders}

The exposure of interest was prescribing of antihypertensives as an FDC compared with prescribing of individual antihypertensives separately.

To adjust for potential differences between prescriptions containing FDCs and separate components, the following potential confounders were included: the age and sex of the patient, and the type of antihypertensive (ACE inhibitor, ARB or beta blocker) and total antihypertensive dosage dispensed (expressed using the WHO classification of defined daily dosages or DDD). We categorised based on drug class for adjustment due to the large number of individual drug combinations. To account for potential differences in cardiovascular comorbidities, the analysis adjusted for the total numbers of items on the same prescription claim from each of the following classes (defined by WHO Anatomical Therapeutic Classification codes):

- Other antihypertensives (C02, C03, C07, C08, C09).

- Lipid-lowering agents (C10).

- Anticoagulants/antiplatelets (B01).

- Antidiabetic agents (A10).
- Other cardiovascular agents (C01, C04, C05).

Lastly, the number of other items (excluding the above categories) on the prescription claim was also adjusted for. Previous research on the relationship between FDCs and adherence has similarly adjusted for coprescribing of other medicines. ${ }^{16} 17$

\section{Statistical methods}

Analyses were all conducted at the level of the pharmacy claim (ie, prescription). Descriptive statistics are presented for included dispensed prescriptions and separately for FDC and FC prescriptions. Differences between the FDC and FC groups were assessed by calculating standardised mean differences (SMD) for each variable, which are independent of sample size.

Regression models were fitted for therapeutic duplication as the binary outcome variable, and combination status (FDC vs FC) as the exposure variable of interest. This analytical approach was then repeated for the secondary outcome of presence of a potentially serious DDI. The non-independence of repeated dispensings to individuals was accounted for by estimating robust standard errors. Generalised linear models using the Poisson distribution were fitted to produce unadjusted and adjusted estimates of the relative risk (RR) of therapeutic duplication, with 95\% CIs. Age, sex, antihypertensive type and DDDs in the FDC/ FC, and the numbers of other prescription items mentioned above were adjusted for.

A propensity score (representing the probability of an FDC being prescribed) was generated by fitting a logistic regression model including all of the prescription and patient characteristics as covariates. This was used to match FDC and FC prescriptions using a calliper of 0.2 of the SD of the logit of the propensity score. ${ }^{18}$ This allowed for the absolute risk difference (ARD) between the FDC and FC prescriptions for therapeutic duplication and DDI to be calculated in both crude and propensity score-matched analyses. By taking the reciprocal of these, we also determined the numbers needed to treat to cause harm (NNTH; ie, the number of patients who need to be prescribed an FDC rather than an FC to cause one additional case of therapeutic duplication/DDI). To assess the impact of residual covariate imbalance, the propensity score-matched regression was repeated with double adjustment for covariates with an SMD of $>0.10$ after matching. ${ }^{19}$ Different approaches to propensity score adjustment were also conducted as a sensitivity analysis, that is, adjusting for propensity score (crude, trimmed, quintiles) in the regression alone or with other covariates.

To assess the robustness of the results and impact of potential residual confounding, sensitivity analysis was conducted to assess the magnitude of effect of residual confounding that would fully explain the observed results. ${ }^{20}$

\section{RESULTS}

\section{Descriptive statistics}

A total of 459465 prescriptions, issued to 49283 patients, were included in this study, 307833 (67\%) containing an FDC and the remaining 151632 (33\%) containing an equivalent FC. Descriptive statistics for included prescriptions are provided in table 1. Fifty per cent of FDC prescriptions were for women, with a mean age of 67.1 (SD 12.5) years, while for FCs, $48.1 \%$ of prescriptions were for women and the mean age was 70.1 (12) years. Prescriptions for FDCs involved an ARB more frequently and an ACE inhibitor or beta blocker less frequently compared with FC prescriptions. FDCs had higher doses of antihypertensives than FCs, although these prescriptions were less likely to contain another coprescribed 
Table 1 Descriptive characteristics of included prescription claims*

\begin{tabular}{|c|c|c|c|}
\hline & $\mathrm{FC}(\mathrm{n}=151632)$ & FDC $(n=307833)$ & Standardised mean difference \\
\hline \multicolumn{4}{|l|}{ Age of prescription recipient (years) } \\
\hline Mean (SD) & $70.1(12.0)$ & $67.1(12.5)$ & 0.021 \\
\hline Female prescription recipient, $\mathrm{n}(\%)$ & $72968(48.1)$ & $154037(50.0)$ & 0.077 \\
\hline \multicolumn{4}{|l|}{ Antihypertensive typet, $\mathrm{n}(\%)$} \\
\hline Beta blocker & $4630(3.1)$ & $1294(0.4)$ & 1.246 \\
\hline ACE inhibitor ( \pm beta blocker) & $69671(45.9)$ & $108671(35.3)$ & 0.553 \\
\hline ARB ( \pm beta blocker or ACE inhibitor) & $77331(51.0)$ & $197868(64.3)$ & 0.446 \\
\hline \multicolumn{4}{|c|}{ Total number of antihypertensive DDDs prescribed } \\
\hline Mean (SD) & $87.3(31.1)$ & $88.7(26.1)$ & 0.017 \\
\hline Any other BP drugs, $\mathrm{n}(\%)$ & $67356(44.4)$ & $104454(33.9)$ & 0.294 \\
\hline Any other CV drug, $\mathrm{n}(\%)$ & $9020(5.9)$ & $12625(4.1)$ & 0.308 \\
\hline Any antiplatelet/anticoagulant, $\mathrm{n}(\%)$ & $72333(47.7)$ & $105838(34.4)$ & 0.450 \\
\hline Any cholesterol drug, $\mathrm{n}(\%)$ & $82553(54.4)$ & $138484(45.0)$ & 0.359 \\
\hline Any diabetic drug, $\mathrm{n}(\%)$ & $18036(11.9)$ & $25090(8.2)$ & 0.248 \\
\hline \multicolumn{4}{|l|}{ Number of other items on prescription } \\
\hline Median (IQR) & $2(1-4)$ & $2(0-3)$ & 0.064 \\
\hline
\end{tabular}

*Issued to 49283 patients.

†Prescriptions containing $\geq 1$ antihypertensive type were categorised in a hierarchy as follows: 1090 ARB prescriptions also contained a combination involving an ACE inhibitor and 34 involving a beta blocker. Ninety-six ACE inhibitor prescriptions also contained a combination involving a beta blocker.

$A R B$, angiotensin II receptor blocker; BP, blood pressure; CV, cardiovascular; DDD, defined daily dosage; FC, free combination; FDC, fixed-dose combination.

medication to treat cardiovascular disease and contained fewer other prescription items.

\section{Therapeutic duplication and DDIs}

Of all included prescriptions, $0.8 \%$ had an instance of therapeutic duplication on the same prescription claim, most often relating to calcium channel blockers, and $10.6 \%$ contained a potentially serious DDI relating to a combination ingredient (see tables 2 and 3). Calcium channel blockers were the most commonly implicated of the antihypertensive agents in both duplications and interactions, with amlodipine and simvastatin being the most frequently

Table 2 Prevalence of therapeutic duplication and regression analyses for risk of duplication

\begin{tabular}{|c|c|c|}
\hline & $\begin{array}{l}\text { FC } \\
(n=151632)\end{array}$ & $\begin{array}{l}\text { FDC } \\
(n=307833)\end{array}$ \\
\hline Therapeutic duplication, $\mathrm{n}(\%)$ & $918(0.61)$ & $2723(0.88)$ \\
\hline ARB & $57(0.04)$ & $404(0.13)$ \\
\hline ACE inhibitor & $103(0.07)$ & $201(0.07)$ \\
\hline Beta blocker & $129(0.09)$ & $128(0.04)$ \\
\hline \multirow[t]{2}{*}{ Calcium channel blocker } & $629(0.41)$ & $2014(0.65)$ \\
\hline & Relative risk $(95 \% \mathrm{Cl})$ & $P$ values \\
\hline Unadjusted regression & 1.46 (1.17 to 1.83$)$ & 0.001 \\
\hline Adjusted regression* & 2.10 (1.67 to 2.65$)$ & $<0.001$ \\
\hline $\begin{array}{l}\text { Propensity score-matched } \\
\text { regressiont }\end{array}$ & $2.29(1.81$ to 2.90$)$ & $<0.001$ \\
\hline $\begin{array}{l}\text { As above, adjusting for covariates } \\
\text { with an SMD }>0.10\end{array}$ & 2.24 (1.77 to 2.83$)$ & $<0.001$ \\
\hline \multicolumn{3}{|c|}{$\begin{array}{l}\text { *Adjusted for patient age and gender, type of combination product (ARB, ACE } \\
\text { inhibitor or beta blocker), and total number of antihypertensive defined daily } \\
\text { dosages (DDD) in the FC/FDC, number of other antihypertensive prescription } \\
\text { items, number of other cardiovascular drugs, number of antiplatelet/anticoagulant } \\
\text { drugs, number of cholesterol drugs, number of diabetes drugs and number of other } \\
\text { prescribed items. } \\
\text { †Propensity score generated from logit model including all covariates adjusted for } \\
\text { in *. } \\
\text { ARB, angiotensin II receptor blockers; FC, free combination; FDC, fixed-dose } \\
\text { combination; SMD, standardised mean differences. }\end{array}$} \\
\hline
\end{tabular}

occurring individual interaction (in $40.5 \%$ of all prescriptions with an interaction, online supplementary table S3).

The crude RR of drug duplication on FDC prescriptions compared with FC prescription was 1.46 (95\% CI 1.17 to 1.83 , ARD $0.28 \%$; NNTH 358), and the RR was higher after adjusting for prescription and patient characteristics (table 2). A propensity score match was successful for $97 \%$ of FC prescriptions (online supplementary figure S1 and table S4) and in propensity score-matched regression, the RR of duplication was 2.29 (95\% CI 1.81 to 2.90 ; ARD 0.75\%; NNTH 133). The magnitude did not vary substantially regardless

Table 3 Prevalence of potentially serious drug-drug interactions and regression analyses for risk of interaction

\begin{tabular}{|c|c|c|}
\hline & $\begin{array}{l}\text { FC } \\
(n=151632)\end{array}$ & $\begin{array}{l}\text { FDC } \\
(n=307833)\end{array}$ \\
\hline Drug-drug interaction, n (\%) & 20206 (13.33) & $28477(9.25)$ \\
\hline ARB & $4905(3.23)$ & $8706(2.83)$ \\
\hline ACE inhibitor & $5114(3.37)$ & $7623(2.48)$ \\
\hline Beta blocker & $78(0.05)$ & $43(0.01)$ \\
\hline \multirow[t]{2}{*}{ Calcium channel blocker } & $12775(8.43)$ & $16378(5.32)$ \\
\hline & Relative risk $(95 \% \mathrm{Cl})$ & $P$ values \\
\hline Unadjusted regression & 0.69 (0.66 to 0.73$)$ & $<0.001$ \\
\hline Adjusted regression* & 1.00 (0.94 to 1.05$)$ & 0.934 \\
\hline $\begin{array}{l}\text { Propensity score-matched } \\
\text { regressiont }\end{array}$ & 1.09 (1.03 to 1.15$)$ & 0.005 \\
\hline $\begin{array}{l}\text { As above, adjusting for } \\
\text { covariates with an SMD }>0.10\end{array}$ & 1.07 (1.01 to 1.13$)$ & 0.030 \\
\hline
\end{tabular}

${ }^{*}$ Adjusted for patient age and gender, type of combination product (ARB, ACE inhibitor or beta blocker), and total number of antihypertensive defined daily dosages (DDD) in the FC/FDC, number of other antihypertensive prescription items, number of other cardiovascular drugs, number of antiplatelet/anticoagulant drugs, number of cholesterol drugs, number of diabetes drugs and number of other prescribed items.

tPropensity score generated from logit model including all covariates adjusted for in *.

$A R B$, angiotensin II receptor blockers; FC, free combination; FDC, fixed-dose combination; SMD, standardised mean differences. 


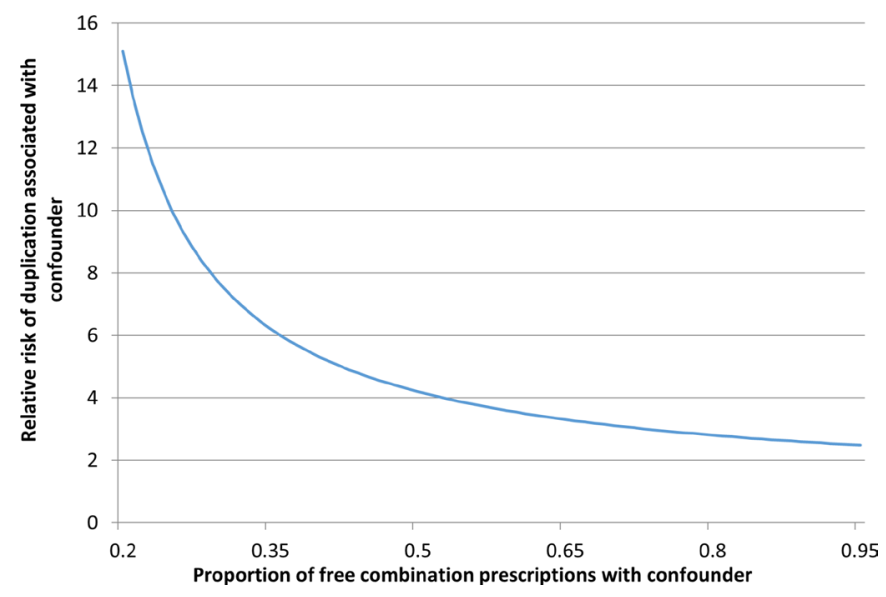

Figure 1 Effect of an unmeasured confounder present in 0.05 of fixed-dose combination (FDC) prescription claims required to explain observed association between FDC prescriptions and duplication.

of how the propensity score was used for adjustment (see online supplementary table S5).

For DDIs (table 3), the risk was lower for FDC prescriptions relative to $\mathrm{FC}$ prescriptions in unadjusted analysis (RR 0.69, $95 \%$ CI 0.66 to 0.73 , ARD $-4.07 \%$; NNTH -25$)$. Adjusting for covariates, the RR was 1.00 (95\% CI 0.94 to 1.05). Propensity score matching yielded an RR of 1.09 (95\% CI 1.03 to 1.15 ; ARD $0.98 \%$; NNTH 101); however, most other methods of propensity score adjustment produced RR estimates that were not statistically significant (online supplementary table S5).

\section{Sensitivity analysis}

Considering the largest effect an unmeasured confounder would need to explain the observed association between FDC prescriptions and therapeutic duplication, a factor occurring in 5\% of FDC prescription would need to have an RR of therapeutic duplication of $7.6,4.2$ or 2.5 if it was present in $30 \%, 50 \%$ or $95 \%$ of FC prescriptions, respectively (see figure 1 ).

\section{DISCUSSION}

This study found that prescriptions for antihypertensive FDCs carry a twofold increased risk of therapeutic duplication compared with the FC ingredients prescribed separately; however, the absolute risk of duplication associated with FDCs was less than $1 \%$. Although the risk of DDIs was higher at approximately $10 \%$, there was no apparent difference between FDC and FC prescriptions.

Although some FDC medications have been flagged as medications which increase the risk of prescribing or dispensing errors, ${ }^{21}$ there appears to be little empirical evidence of this. Much of the literature has focused on assessing the most common types of medication errors, and the drug classes most frequently involved. ${ }^{122}$ By contrast, alternate medications or formulations and their risk of medication errors have received less attention. For instance, oral dosage forms via a monitored dosage system result in fewer administration errors among care home residents. ${ }^{23}$ There are several potential ways that FDCs may increase risk of medication errors. Greater than two strengths of two ingredients in an FDC will result in more products listed in an electronic record system or on a pharmacy shelf, increasing the risk of selection errors. ${ }^{12}$ Dosing errors may result from FDCs containing two ingredients of the same strengths, for example, combinations of amlodipine 5 or $10 \mathrm{mg}$ and perindopril arginine
5 or $10 \mathrm{mg}$. These action-based errors, that is, discordance between the intended product to be prescribed/dispensed and the actual action, could not be identified using our data source. ${ }^{22}$ Instead, these pharmacy claims were used to evaluate the knowledge-based errors of duplication and DDIs, for which there are a number of plausible mechanisms. As marketing of FDCs often results in patent extension of off-patent drugs, brand names are more likely to be used for FDCs. ${ }^{24}$ Brand names are more often confused with another at the prescribing/dispensing stage than generic or international non-proprietary names (INN). ${ }^{925}$ Moreover, the information provided by the INN suffix on a drug's therapeutic class (ie, -sartan indicating an ARB) is lost when prescribing a brand name. ${ }^{9}$ Hence, healthcare professionals may be less likely to identify a drug interaction or duplication if they do not recognise the type of drug an FDC contains. ${ }^{9-11}$

Knowledge-based errors such as therapeutic duplication or drug interactions may be mitigated through the use of computer-based clinical decision support systems (CDSS). ${ }^{26}$ The majority of prescribing/dispensing systems incorporate CDSS capable of detecting instances of therapeutic duplication and DDIs. ${ }^{27}$ It is possible that implementation of duplication/interaction checking may be less comprehensive for combination products if software is only capable of cross-referencing one active ingredient per medication. A lack of reference to the calcium channel blocker component of combination products in the software drug product file may explain the increased risk of therapeutic duplication. However, due to the proprietary nature of the prescribing/dispensing software systems used in Ireland, we are unable to determine their capability for cross-referencing products with multiple ingredients. Even when detection is effective, CDSS alerts ought to be implemented appropriately to minimise alert fatigue and ensure such medication issues are acted on where necessary. This is an important consideration in the development and implementation of electronic health records, particularly given the increasing use of combination products across therapeutic domains. In addition, increased generic prescribing and dispensing (ie, using INN) may also reduce medication errors. In a simulated before and after study of generic labelling of medications, FDCs were one of just two circumstances where errors decreased. ${ }^{28}$ When patients and doctors are considering an FDC, it is important to assess the potential risks and benefits. Although use of FDCs can reduce pill burden, ${ }^{17}$ the potential impact on medication adherence is complex. A lower pill burden can reduce regimen complexity; however, this is only one of over 700 potential factors found to be associated with non-adherence, ${ }^{29}$ and so any potential benefit of FDCs is dependent on the factors contributing to non-adherence in each individual patient's case. FDCs could have a negative impact on some aspects of adherence, for example, unnecessarily high doses due to inflexible combinations could result in treatment discontinuation (or non-persistence) due to adverse effects. FDCs can also provide economic benefits. ${ }^{16} 24$ These benefits should however be weighed against the potential for iatrogenic harm due to medication errors. Even if the risk is low, medication errors can cause a substantial burden of patient harm, morbidity and mortality. ${ }^{13}$

This study has a number of strengths and limitations. This is a large analysis which used the PCRS database, the most comprehensive source of medication prescribing and dispensing information in Ireland, capturing approximately $40 \%$ of the population. ${ }^{15}$ As this is an observational study, there may be residual confounding due to unmeasured factors which predict both whether antihypertensive medications 
are prescribed in combination and therapeutic duplication/ drug interactions, such as whether a prescriber uses CDSS. Due to the retrospective nature of this study we could not collect data on these additional factors. However, the results were robust to various methods of adjustment for measured confounders, and we quantified the magnitude of confounding due to unmeasured factors that would alter the findings. Other potential action-based medication errors (ie, selecting a different product than intended) could not be evaluated. In addition, these data include only medications dispensed on the GMS scheme. ${ }^{15}$ The scheme population is not representative of the general population (tending to be older and more socioeconomically deprived), and so the observed association may differ in other populations. We were unable to adjust for clustering of prescriptions at the prescriber level, and so the width of the CIs may be underestimated. However, some of the prescriber-level effects are likely to be accounted for in our adjustment for patient-level clustering. There may be clinical awareness and rationale for the duplications and interactions detected in this study, but regardless of whether these were intentional, FDCs appear to be associated with marginally higher therapeutic duplication. The definition of therapeutic duplication used did not include cases where the same active ingredient was prescribed in two different medications as this has the potential to be appropriate and intentional, thus our results represent a conservative estimate of the extent of therapeutic duplication. Similarly, by excluding DDIs that relate solely to hypotension where a prescriber may have titrated the antihypertensive doses to account for such an interaction, we have aimed to reduce misclassification of medication errors.

Compared with prescribing antihypertensive drugs as separate medications, prescriptions for FDC medications were associated with greater risk of therapeutic duplication but not DDIs. The absolute risk is small and should be considered in light of potential benefits of FDCs to patients. If FDCs and polypills are to be implemented more widely as has been proposed, ${ }^{30}$ it is important to consider how their use may influence prescribing quality and risk of medication errors, and thus medication safety. Systems to reduce medication errors, such as CDSS, should be evaluated to ensure they are functional for both FDC and single ingredient medications.

\section{Key questions}

\section{What is already known on this subject?}

- Fixed-dose combinations or polypills containing multiple ingredients in a single medication are increasingly common in managing cardiovascular disease and can improve medication adherence.

\section{What might this study add?}

- This study found the prescriptions for fixed-dose combinations were associated with a higher chance of therapeutic duplication compared with the equivalent ingredients prescribed separately, but not with drug-drug interactions.

\section{How might this impact on clinical practice?}

- Healthcare professionals or patients who use fixed-dose combinations may exercise caution and vigilance with these medications in order to minimise medication errors and patient harm.

\section{Twitter@FrankMoriarty@HRBPrimaryCare}

Acknowledgements We acknowledge the Primary Care Reimbursement Service for providing the administrative data used in this study.

Contributors FM conceived and designed the study. $\mathrm{KB}$ acquired the data and FM conducted the analysis. FM, KB and TF interpreted the data. FM drafted the manuscript and all authors revised this critically and approved the final version.

Funding This research was supported by the Health Research Board (HRB) in Ireland through the HRB Centre for Primary Care Research (grant number HRC/2014/1). KB was supported by the HRB (grant number RL/15/1579).

\section{Competing interests None declared.}

Patient consent Not required.

Provenance and peer review Not commissioned; externally peer reviewed. Data sharing statement There are no additional data available from this study.

Open access This is an open access article distributed in accordance with the Creative Commons Attribution Non Commercial (CC BY-NC 4.0) license, which permits others to distribute, remix, adapt, build upon this work non-commercially, and license their derivative works on different terms, provided the original work is properly cited, appropriate credit is given, any changes made indicated, and the use is non-commercial. See: http://creativecommons.org/licenses/by-nc/4.0/.

\section{REFERENCES}

1 European Medicines Agency Committee for Medicinal Products for Human Use (2017) Guideline on Clinical Development of Fixed Combination Medicinal Products. London, UK.

2 Huffman MD, Xavier D, Perel P. Uses of polypills for cardiovascular disease and evidence to date. Lancet 2017;389:1055-65.

3 Bangalore S, Kamalakkannan G, Parkar S, et al. Fixed-dose combinations improve medication compliance: a meta-analysis. Am J Med 2007:120:713-9.

4 Thom S, Poulter N, Field J, et al. Effects of a fixed-dose combination strategy on adherence and risk factors in patients with or at high risk of CVD: the UMPIRE randomized clinical trial. JAMA 2013;310:918.

5 Gradman AH, Basile JN, Carter BL, et al. Combination therapy in hypertension. J Am Soc Hypertens 2010;4:90-8.

6 Barnett K, Mercer SW, Norbury M, et al. Epidemiology of multimorbidity and implications for health care, research, and medical education: a cross-sectional study. Lancet 2012;380:37-43.

7 Farrell B, French Merkley V, Ingar N. Reducing pill burden and helping with medication awareness to improve adherence. Can Pharm J 2013;146:262-9.

8 Garjón J, Saiz LC, Azparren A, et al. First-line combination therapy versus first-line monotherapy for primary hypertension. Cochrane Database Syst Rev 2017;1:CD010316.

9 Aronson JK. Medication errors resulting from the confusion of drug names. Expert Opin Drug Saf 2004;3:167-72.

10 Bramley DE. The ability of anaesthetists to identify generic medications from trade names. Anaesth Intensive Care 2009;37:624-9.

11 Schwab M, Oetzel C, Mörike K, et al. Using Trade Names. Arch Intern Med 2002:162:1065.

12 Bobb A, Gleason K, Husch M, et al. The Epidemiology of Prescribing Errors. Arch Intern Med 2004;164:785.

13 Karnon J, McIntosh A, Dean J, et al. Modelling the expected net benefits of interventions to reduce the burden of medication errors. J Health Serv Res Policy 2008;13:85-91.

14 von Elm E, Altman DG, Egger M, et al. The Strengthening the Reporting of Observational Studies in Epidemiology (STROBE) statement: guidelines for reporting observational studies. PLoS Med 2007;4:e296.

15 Sinnott SJ, Bennett K, Cahir C. Pharmacoepidemiology resources in Ireland-an introduction to pharmacy claims data. Eur J Clin Pharmacol 2017;73:1449-55.

16 Hess G, Hill J, Lau H, et al. Medication Utilization Patterns and Hypertension-Related Expenditures among Patients Who Were Switched from Fixed-Dose To FreeCombination Antihypertensive Therapy. P T 2008:33:652-66.

17 Bahiru E, de Cates AN, Farr MR, et al. Fixed-dose combination therapy for the prevention of atherosclerotic cardiovascular diseases. Cochrane Database Syst Rev 2017;3:CD009868

18 Austin PC. Optimal caliper widths for propensity-score matching when estimating differences in means and differences in proportions in observational studies. Pharm Stat 2011;10:150-61.

19 Nguyen TL, Collins GS, Spence J, et al. Double-adjustment in propensity score matching analysis: choosing a threshold for considering residual imbalance. BMC Med Res Methodol 2017;17:78

20 Schneeweiss $S$. Sensitivity analysis and external adjustment for unmeasured confounders in epidemiologic database studies of therapeutics. Pharmacoepidemiol Drug Saf 2006;15:291-303. 
21 Irish Medication Safety Network. Briefing Document on Sound-Alike Look-Alike Drugs (SALADS), 2010.

22 Aronson JK. Medication errors: what they are, how they happen, and how to avoid them. QJM 2009;102:513-21.

23 Alldred DP, Standage C, Fletcher O, et al. The influence of formulation and medicine delivery system on medication administration errors in care homes for older people. BMJ Qual Saf 2011;20:397-401.

24 Hao J, Rodriguez-Monguio R, Seoane-Vazquez E. Fixed-dose combination drug approvals, patents and market exclusivities compared to single active ingredient pharmaceuticals. PLoS One 2015;10:e0140708.

25 Berman A. Reducing medication errors through naming, labeling, and packaging. $J$ Med Syst 2004;28:9-29.
26 Wolfstadt JI, Gurwitz JH, Field TS, et al. The effect of computerized physician order entry with clinical decision support on the rates of adverse drug events: a systematic review. J Gen Intern Med 2008;23:451-8.

27 Roblek T, Vaupotic T, Mrhar A, et al. Drug-drug interaction software in clinical practice: a systematic review. Eur J Clin Pharmacol 2015;71:131-42.

28 Garcia BH, Elenjord R, Bjornstad C, et al. Safety and efficiency of a new generic package labelling: a before and after study in a simulated setting. BMJ Qual Saf 2017:26:817-23.

29 Kardas P, Lewek P, Matyjaszczyk M. Determinants of patient adherence: a review of systematic reviews. Front Pharmacol 2013;4:91.

30 Webster R, Castellano JM, Onuma OK. Putting polypills into practice: challenges and lessons learned. Lancet 2017;389:1066-74. 